CentroSur

Social Science Journal

\section{Ausencia de los derechos de los estudiantes de la Universidad Nacional de Loja: estudio de caso sobre la calidad de vida y la vulnerabilidad social}

\begin{abstract}
Absence of the rights of the students of the National University of Loja: case study on quality of life and social vulnerability
\end{abstract}

Centro Sur.

Social Science Journal Julio - Diciembre Vol 4 No 2 http://centrosureditorial.com/ind ex.php/revista elSSN: 2600-5743 revistacentrosur@gmail.com Recepción: 08 abril 2019 Aprobación 15 junio 2019 Pag 205 - 222

Atribución/ReconocimientoNoComercial-Compartirlgual 4.0 Licencia Pública Internacional CC BY-NC-SA 4.0 https://creativecommons.org/lice nses/by-nc-sa/4.0/legalcode.es

\author{
Beatriz Carrión - Berrú ${ }^{1}$ \\ Betti Reyes - Masa ${ }^{2}$ \\ Jorge Calle - Iñiguez ${ }^{3}$ \\ Jefferson Sanchez - Ruiz ${ }^{4}$
}

\section{Resumen}

Identificar los indicadores de vulnerabilidad para prevenir la exclusión de los miembros de la comunidad universitaria de la Universidad Nacional de Loja, se remite esencialmente a la situación de riesgo a la que se ve expuesta la comunidad universitaria ante cambios en las condiciones del entorno, sobre el tema es posible encontrar estudios de índole muy diversa que tiene su denominador común en el énfasis sobre situaciones de perjuicio potencial. Se realizó una medición que estuvo íntimamente relacionada con las dimensiones de la escala analizada en su contexto social, económico y cultural, desde un enfoque de derechos, en donde el ser humano tiene una mirada integral; se generaron datos a través de la aplicación del método de GENCAT y se presenta un análisis de los resultados de bienestar físico, derechos, autodeterminación, inclusión social caso de la UNL en estudiantes de las 31 carreras

\footnotetext{
${ }^{1}$ Magíster en Desarrollo Comunitario. Universidad Nacional de Loja, Loja, Ecuador. Orcid: /0000-00020575-1757. Email: celsa.carrion@unl.edu.ec. Google scholar:

https://scholar.google.es/citations?view_op=list_works\&hl $=$ es\&user $=r$ DiPvsMAAAAJ

2 Doctora en Trabajo Social. Universidad Nacional de Loja, Loja, Ecuador. Orcid: /0000-0003-3135-8606. Email: betti.reyes@unl.edu.ec Google scholar: https://scholar.google.es/citations?view_op=list_works\&hl =es\&user=dZPtgacAAAAJ
${ }^{3}$ Magíster en Finanzas. Universidad Nacional de Loja. Loja, Ecuador. Orcid: /0000-0002-9679-0590. Email: jorge.calle@unl.edu.ec.
${ }^{4}$ Magíster en Innovación de la gestión turística. Universidad Técnica Particular de Loja, Universidad Nacional de Loja. ORCID: 0000-0003-3135-8606. Google scholar: Email: jjsanchez33@utpl.edu.ec.
https://scholar.google.es/citations?user=0ZahmqQAAAAJ \&hl=es


presenciales y 9 de la modalidad a distancia.

Palabras Clave: calidad de vida,
derechos, vulnerabilidad,
autodeterminación personal.

\section{Abstract}

Identifying vulnerability indicators to prevent the exclusion of members of the university community of the National University of Loja, essentially refers to the risk situation to which the university community and people are exposed to changes in environmental conditions, On the subject it is possible to find very diverse studies that have their common denominator in the emphasis on situations of potential harm. A measurement was made that was closely related to the dimensions of the scale analyzed in its social, economic and cultural context, from a rights perspective, where the human being has an integral view; Data were generated through the application of the GENCAT method and an analysis of the results of Physical Wellbeing, Rights, SelfDetermination, Social Inclusion of the UNL case in students of the 31 face-to-face careers and 9 of the distance modality is presented.

Key words: quality of life, rights, vulnerability, personal self-determination

\section{Introducción}

La vulnerabilidad que atraviesan los integrantes de los diferentes estamentos universitarios, es un problema de investigación en cuanto a lo científico debido a que se encuentra "invisibilizada" inclusive es ausente su debate, lo que implica definir y tomar conciencia de las condiciones de vulnerabilidad que atraviesa el individuo, grupo y comunidad; al respecto cabe acotar el marco teórico de ( Labrunée \& Gallo , 2005), "el concepto de vulnerabilidad hace referencia a la potencialidad de sufrir daños a raíz de fenómenos o acontecimientos de orden externo". Dichos autores proponen dos extremos: la protección total o invulnerabilidad absoluta y la completa indefensión; y entre estos extremos una gama de situaciones intermedias.

La idea de vulnerabilidad social remite esencialmente a la situación de riesgo a la que se ven expuestas comunidades, familias y personas ante cambios en las condiciones del entorno, tal que el concepto de vulnerabilidad social es relativamente nuevo y se halla aún en plena etapa de gestación teórica y metodológica, por lo que en la bibliografía existente sobre el tema es posible encontrar estudios de índole muy diversa que tiene su denominador común en el énfasis sobre situaciones de perjuicio potencial.

Asimismo, siguiendo autores como ( Labrunée \& Gallo , 2005), mencionan el carácter intrínsecamente complejo del fenómeno que permite una aproximación al mismo a partir de las distintas dimensiones que asume; así puede hablarse de vulnerabilidad social poniendo énfasis en el aspecto sociodemográfico, en las problemáticas 
vinculadas a la inserción laboral, o en las posibilidades o restricciones que se presentan a partir de los recursos de que disponen los hogares de los miembros de la comunidad universitaria. Pero en todos los casos, se destacan dos características fundamentales que hacen a la esencia del concepto de vulnerabilidad social y que lo diferencian de otras categorías analíticas. En primer lugar, se destaca el carácter dinámico y multidimensional del problema, en contraste con otras concepciones que dan cuenta de una situación estática de malestar social, como pobreza y exclusión.

A raíz de un proyecto de investigación ejecutado en el año 2019 en la comunidad universitaria sobre este tema, existe una oportunidad debido a que la Unidad de Bienestar Universitario, cuenta con información social y económica dispersa, en lo que se relaciona con su población estudiantil, sus docentes, los servidores administrativos, trabajadores, debido a que las diferentes instancias administrativas requieren y custodian información que implica la construcción de métricas de forma separada para fines específicos, así la Unidad de Talento Humano mantiene información laboral, la Dirección de docencia mantiene información académica, y particularmente el departamento de Bienestar Universitario también tiene información relacionada con riesgos laborales, incidentes de salud de las/los estudiantes, de trabajadores y de docentes; la cual se origina en la permanente atención y contingencias que actualmente brinda la unidad; pero la información disponible no adecua datos que permitan prevenir contingentes, ampliar coberturas, mejorar servicios, establecer políticas, y sobre todo coadyuvar en el aumento de la calidad de vida de los miembros de la comunidad universitaria.

Así pues, y muy a pesar de los esfuerzos y atenciones realizadas por "Bienestar Estudiantil", se observa madres solteras, jóvenes embarazadas, jóvenes con síntomas de alcoholismo, noticias de microtráfico en los predios de la Universidad; en lo que corresponde a "Bienestar docente, administrativo y trabajadores" las ausencias que se dan por temas de enfermedad, calamidad doméstica y otros aspectos, dan cuenta que existen situaciones que la Universidad desconoce sobre la comunidad universitaria en su parte humana; asimismo, el desconocimiento de normativas laborales como es el "comité paritario" entre otras; podrían estar generando exclusiones (como efecto de la vulnerabilidad) y por tanto injusticias que redundan en la calidad de vida del ser humano y el equilibrio y cohesión interna de los miembros de la comunidad universitaria.

Más allá de las problemáticas expuestas, y en el marco de la discusión sobre las potencialidades para remediar el problema surge la oportunidad de construir instrumentos para proveer antecedentes útiles para el diseño y la implementación de políticas sociales y sobre todo investigar las situaciones de vulnerabilidad social que 
podría atravesar los miembros de la comunidad y sobre todo las/los estudiantes. Además, hacer de esta información la base de indicadores de usos y fuentes de activos ayudaría a la construcción de una mejor comunidad universitaria.

La Universidad Nacional de Loja, propone formar una ciudadanía con profesionales de una práctica coherente, vivenciada y reflexionada como aspecto central del Bienestar Universitario, en la actualidad se requiere modificar, tanto sustantiva como formalmente, aquellos aspectos limitantes de la misión y la visión de la UNL que están bajo la competencia de la Dirección de Bienestar Universitario; además, responder a las expectativas y necesidades presentes y futuras de la población universitaria (Esthela \& Betti, 2010).

Asimismo, ante la necesidad de prevalecer sobre las exigencias de la evaluación externa, la Universidad requiere construir evidencias que demuestren que las actividades y servicios generales que administra, están orientados a la formación integral y al bienestar de los miembros de la comunidad universitaria.

Se suma a ello que, entre las estrategias contenidas en el Quinto Plan de Desarrollo Institucional 2013-2018, la estrategia G.1.1 enunciada como fortalecimiento de la gestión en los diferentes ámbitos del quehacer institucional contiene como su primer Proyecto: Construcción participativa de la nueva estructura organizacional de la UNL; las autoridades actuales elegidas para el período 2019-2023 están llevando a cabo el proceso de construcción y ejecución del Plan Estratégico de Desarrollo Institucional de todos los estamentos con la finalidad de fortalecer la participación y disminuir la exclusión social generando vínculos colectivos entre estudiantes, docente y personal administrativo (UNL, 2018).

Para el proceso de mejoramiento del Departamento de Bienestar Universitario, se debe desarrollar consecuentemente una política amplia y consensuada con todos los estamentos universitarios, prioritaria y de ejecución inmediata. De ahí que es inaplazable que el Departamento se relacione plenamente con la comunidad universitaria, sea parte de ella como un organismo del sistema interno de manera integral e integrada.

Por ello, calidad de vida se ha convertido en uno de los conceptos internacionalmente más identificados con el movimiento de avance, innovación y cambio en las prácticas profesionales y en los servicios, permitiendo promover actuaciones en los aspectos de la persona, de la organización y del sistema social por lo que para quienes trabajamos en lo social es de vital importancia; así, se utilizó la escala de GENCAT, que es un instrumento de evaluación objetiva de acuerdo con los avances realizados sobre el modelo multidimensional de calidad de vida propuesto por Schalock y 
Verdugo (2002/2003), que actualmente cuentan con gran aceptación internacional. La escala de GENCAT fue pensada para ser utilizada como instrumento para la mejora continua de los servidores sociales, con la intención de que los planes de atención y apoyo que estos realizan sean más personalizados y adecuados a las necesidades actuales y futuras de los y las usuarias.

\section{La dimensión social de la calidad de vida}

La Dimensión social se refiere a la habilidad de relacionarse con otras personas. Se la obtiene mediante una comunicación que implique una escucha activa, así como la puesta en práctica de actitudes asertivas y empáticas con los demás.

Definición según la Organización Mundial de la Salud según la OMS, la calidad de vida es la percepción que un individuo tiene de su lugar en la existencia, en el contexto de la cultura y del sistema de valores en los que vive y en relación con sus objetivos, sus expectativas, sus normas, sus inquietudes.

Calidad de vida es un concepto que hace alusión a varios niveles de generalización pasando por sociedad, comunidad, hasta el aspecto físico y mental, por lo tanto, el significado de calidad de vida es un complejo y contando con definiciones desde sociología, ciencias políticas, medicina, estudios del desarrollo, etc. Este concepto internacionalmente más identificados con el movimiento de avance, innovación y cambio en las prácticas profesionales y en los servicios, permitió promover actuaciones en los aspectos de la persona, de la organización y del sistema social por lo que para quienes trabajamos en lo social es de vital importancia así pues se utilizó la escala de GENCAT, que es un instrumento de evaluación objetiva de acuerdo con los avances realizados sobre el modelo multidimensional de calidad de vida propuesto por Schalock y Verdugo (2002/2003), que actualmente cuentan con gran aceptación internacional. La escala de GENCAT fue pensada para ser utilizada como instrumento para la mejora continua de los servidores sociales, con la intención de que los planes de atención y apoyo que estos realizan sean más personalizados y adecuados a las necesidades actuales y futuras de los y las usuarias.

Identificación de indicadores de vulnerabilidad, una posibilidad para el cambio de una realidad social con enfoque de derechos

En forma específica se considera que se precisa identificar una serie de indicadores que permitan tener información de quienes conforman la comunidad universitaria para que dicha Unidad tenga insumos para cumplir con reorientar las funciones y actividades que hasta ahora ha venido ejecutando la Dirección de Bienestar Estudiantil, de manera que su servicio llegue a todos los estamentos universitarios profesores, administrativos, trabajadores jubilados, estudiantes y profesionales 
formados por la UNL haciendo que tal reorientación centre los procesos participativos de dichos sujetos y se traduzcan en respuestas efectivas ante las problemáticas.

El término de vulnerabilidad es muy amplio, hace referencia a la potencialidad de sufrir daños a raíz de fenómenos externos y los autores Labrunée y Gallo mencionan entre la protección total o invulnerabilidad absoluta y la completa indefensión existe una gama de situaciones intermedias que definen un gradiente de vulnerabilidad que está en función de las posibilidades de hacer frente a la agresión externa y es ahí donde la presente investigación realizó su aporte de acuerdo a los diferentes actores de la Unidad de Bienestar Universitario. El término de vulnerabilidad social, sin embargo, se realizará un acercamiento al riesgo de familias y de comunidad como también se abordará el intento por comprender la vulnerabilidad sociodemográfica, vulnerabilidad de ingresos o transformaciones económicas, vulnerabilidad laboral, entre otras que durante la investigación se crea la necesidad de análisis.

De esta manera la vulnerabilidad social busca establecer relaciones de causalidad múltiple, procurando identificar las condiciones que refuerzan la reproducción de los procesos de deterioro del nivel de vida de los actores de la investigación y es ahí donde pretendemos identificar los indicadores que nos darán la realidad de lo que ocurre con los miembros de quienes conforma la Unidad de Bienestar Universitario.

La medición de la vulnerabilidad requiere de un examen sincronizado de los activos de las personas (o de los hogares) y de los requerimientos de acceso a las estructuras de oportunidades que tienen su fuente en distintos órdenes institucionales básicos. Al atractivo conceptual que tiene una mirada que considera en forma simultánea fenómenos micro y macro sociales, se contraponen las dificultades metodológicas que plantea una medición que requiere información de unidades que corresponden a niveles de análisis diferentes.

En líneas generales el concepto de vulnerabilidad hace referencia a la potencialidad de sufrir daños a raíz de fenómenos o acontecimientos de orden externo. Entre la protección total o invulnerabilidad absoluta y la completa indefensión existe una gama de situaciones intermedias que definen un gradiente de vulnerabilidad que está en función de las posibilidades de hacer frente a la agresión externa ( Labrunée \& Gallo , 2005).

La idea de vulnerabilidad social se enmarca en esta definición, y remite esencialmente a la situación de riesgo, a la que se ven expuestas comunidades, familias y personas ante cambios en las condiciones del entorno. El concepto de vulnerabilidad social es relativamente nuevo y se halla aún en plena etapa de gestación teórica y metodológica, por lo que en la bibliografía existente sobre el tema es posible encontrar 
estudios de índole muy diversa que tiene su denominador común en el énfasis sobre situaciones de perjuicio potencial. Los trabajos pioneros sobre la cuestión versan acerca del riesgo de familias y comunidades ante desastres naturales o situaciones catastróficas como guerras o hambrunas (Longhurst, 1994). Una segunda vertiente aborda el enfoque de la vulnerabilidad en un intento por comprender el impacto de fenómenos socioeconómicos traumáticos sobre las condiciones de vida de las comunidades rurales (Chambers, 1989; Chambers, 1995; Longhurst, 1994; Buchanan-Smith y Maxwell, 1994; Bayliss- Smith, 1991).

El concepto de vulnerabilidad, se asocia a expresiones como daño, fragilidad, indefensión, desamparo, debilidad, se traslapa en varios aspectos como el de la exclusión, pero hace referencia a las condiciones del ser humano, hogares o comunidades que tienen mayor probabilidad de ser afectados de forma negativa y con menor capacidad de respuesta ante cambios del entorno, los cuales los puede conducir a situaciones de pobreza o afectar los pilares que definen la integración social en la actualidad, es decir el empleo, el acceso a los servicios de salud, de educación, de protección social y de vivienda.

El concepto recoge aportes de las ciencias sociales y del comportamiento en escenarios comunitarios, pero tiene una evidente validez para analizar los procesos de cambio y de crisis en el ámbito organizacional-empresarial. Estas categorías deben dar alguna explicación del porqué unas organizaciones y unas personas dan respuestas positivas a pesar de las dificultades con una mayor facilidad y propiedad que otras. Del porqué algunas empresas y algunas personas resurgen de las dificultades fortalecidas y con una mayor contundencia en su accionar y resultados.

Por lo tanto, el concepto de vulnerabilidad se concatena con una propuesta metodológica para medir la calidad de vida y generar planes de intervención social a través de una escala llamada la Escala GENCAT presenta un conjunto de aspectos observables de distintas áreas que conforman la calidad de vida de una persona y que pueden ser objeto de planes de apoyo personalizados por distintos tipos de servicios sociales. Teniendo en cuenta la distinción realizada en los últimos años entre aspectos objetivos y subjetivos, así como la débil concordancia encontrada entre ellos (Arostegui, 2002; Gómez, 2005; Janssen, Schuengel y Stolk, 2005; Olson y Schoeber, 1993; Perry, Felce y Lowe, 2000; Perry y Felce, 2005; Schalock y Felce, 2004; Verdugo, Arias y Gómez, 2006), este instrumento permite la evaluación objetiva de la calidad de vida proporcionando indicadores sensibles al diseño ambiental y de programas por parte de las organizaciones y servicios.

La Escala GENCAT está basada en este modelo multidimensional en el cual existen ocho dimensiones o dominios que cuando se toman en conjunto constituyen el 
concepto de calidad de vida. Entendemos las dimensiones básicas de calidad de vida como "un conjunto de factores que componen el bienestar personal", y sus indicadores centrales como "percepciones, conductas o condiciones específicas de las dimensiones de calidad de vida que reflejan el bienestar de una persona" (Schalock y Verdugo, 2003, p. 34).

A continuación, se presenta una breve definición de cada una de las ocho dimensiones del modelo, así como los indicadores seleccionados para operativizar dichas dimensiones, que han sido fruto de más de una década de investigación nacional e internacional (Gómez, 2005; Gómez, Verdugo y Arias, 2007; Jenaro [et al.], 2005; Keith y Schalock, 2000; Schalock y Keith, 1993; Schalock, 1996, 1997; Schalock y Verdugo, 2002/2003, 2007; Schalock [et al.], 2005; Verdugo, Arias y Martín, 2006; Verdugo, Schalock, Gómez y Arias, 2007; Verdugo, Gómez y Arias, 2007; Schalock y Verdugo, 2007; Verdugo, Arias, Gómez y Schalock, 2008.2009).

Bienestar emocional (BE): hace referencia a sentirse tranquilo, seguro, sin agobios, no estar nervioso. Se evalúa mediante los indicadores: Satisfacción, Autoconcepto y Ausencia de estrés o sentimientos negativos.

Relaciones interpersonales $(\mathrm{RI})$ : relacionarse con distintas personas, tener amigos/as y llevarse bien con la gente (vecinos, compañeros, etc.). Se mide con los siguientes indicadores: Relaciones sociales, Tener amigos claramente identificados, Relaciones familiares, Contactos sociales positivos y gratificantes, Relaciones de pareja y Sexualidad.

Bienestar Material (BM): tener suficiente dinero para comprar lo que se necesita y se desea tener, tener una vivienda y lugar de trabajo adecuados. Los indicadores evaluados son: Vivienda, Lugar de trabajo, Salario (pensión, ingresos), Posesiones (bienes materiales), Ahorros (o posibilidad de acceder a caprichos).

Desarrollo personal (DP): se refiere a la posibilidad de aprender distintas cosas, tener conocimientos y realizarse personalmente. Se mide con los indicadores: Limitaciones/Capacidades, Acceso a nuevas tecnologías, Oportunidades de aprendizaje, Habilidades relacionadas con el trabajo (u otras actividades) y Habilidades funcionales (competencia personal, conducta adaptativa, comunicación).

Bienestar físico (BF): tener buena salud, sentirse en buena forma física, tener hábitos de alimentación saludables. Incluye los indicadores: Atención Sanitaria, Sueño, Salud y sus alteraciones, Actividades de la vida diaria, Acceso a ayudas técnicas y Alimentación. 
Autodeterminación $(A U)$ : decidir por sí mismo y tener oportunidad de elegir las cosas que quiere, cómo quiere que sea su vida, su trabajo, su tiempo libre, el lugar donde vive, las personas con las que está. Los indicadores con los que se evalúa son: Metas y preferencias personales, Decisiones, Autonomía y Elecciones.

Inclusión social (IS): ir a lugares de la ciudad o del barrio donde van otras personas y participar en sus actividades como uno más. Sentirse miembro de la sociedad, sentirse integrado, contar con el apoyo de otras personas. Evaluado por los indicadores: Integración, Participación, Accesibilidad y Apoyos.

Derechos (DE): ser considerado igual que el resto de la gente, que le traten igual, que respeten su forma de ser, opiniones, deseos, intimidad, derechos. Los indicadores utilizados para evaluar esta dimensión son: Intimidad, Respeto, Conocimiento y Ejercicio de derechos.

\section{El enfoque basado en los derechos humanos}

Los derechos iguales e inalienables de los seres humanos establecen las bases para la libertad, la justicia y la paz en el mundo, según la Declaración Universal de Derechos Humanos, adoptada por la Asamblea General de las Naciones Unidas en 1948. El enfoque basado en los derechos humanos se centra en los grupos de población que son objeto de una mayor marginación, exclusión y discriminación. Este enfoque a menudo requiere un análisis de las normas de género, de las diferentes formas de discriminación y de los desequilibrios de poder a fin de garantizar que las intervenciones lleguen a los segmentos más marginados de la población.

Existen elementos de buenas prácticas propios del enfoque basado en los derechos humanos

Los programas ven en el pleno ejercicio de los derechos humanos el fin último del desarrollo.

Las personas son consideradas agentes principales de su propio desarrollo, en lugar de receptores pasivos de productos y servicios.

La participación es a la vez un medio y un objetivo.

Las estrategias deben proporcionar empoderamiento, en lugar de negarlo.

Tanto los resultados como los procesos son supervisados y evaluados.

Los programas se centran en grupos de la población marginados y excluidos.

El proceso de desarrollo enfocado en lo local. 
Los programas tienen como objetivo reducir las desigualdades y empoderar a quienes se han quedado atrás.

El análisis de situación se utiliza para identificar las causas inmediatas, subyacentes y fundamentales de los problemas de desarrollo.

El análisis incluye a todos los grupos de interés, entre ellos, las capacidades del Estado como principal garante de derechos y el papel de otros agentes no estatales.

Las normas de los derechos humanos dirigen la formulación de objetivos, metas e indicadores medibles en la programación.

Los sistemas nacionales de rendición de cuentas deben ser reforzados con el objetivo de garantizar una revisión independiente de desempeño del gobierno así como el acceso a vías de recurso para los sujetos agraviados.

Se promueve el desarrollo y el mantenimiento de alianzas estratégicas con un trabajo en redes.

\section{Derechos frente a necesidades}

Antes de 1997, la mayor parte de los organismos de desarrollo de las Naciones Unidas adoptaban un enfoque en las necesidades básicas: identificaban las exigencias básicas de los beneficiarios y, o bien apoyaban iniciativas para mejorar la prestación de servicios, o bien fomentaban su cumplimiento.

Un enfoque basado en los derechos humanos también busca reforzar las capacidades de los garantes de derechos (normalmente, los gobiernos) de respetar, proteger y garantizar estos derechos. El objetivo de este enfoque es abordar las complejidades del desarrollo desde una perspectiva holística, teniendo en cuenta las conexiones entre las personas y los sistemas de poder o influencia. Por otra parte, también pretende crear una dinámica para la rendición de cuentas.

Habría que destacar una doble necesidad: por un lado, las personas y las comunidades requieren que se las informe acerca de sus derechos y se les permita participar en aquellas decisiones que las afectan; y, por otro, los gobiernos y otros garantes de derechos a menudo requieren asistencia para desarrollar la capacidad, los recursos y la voluntad política necesarios para poder cumplir sus compromisos respecto a los derechos humanos.

El enfoque basado en los derechos tiene que ver no solo con los resultados sino también con el modo en que se lograrán estos resultados. Reconoce a las personas como agentes que intervienen en su propio desarrollo, en lugar de receptores pasivos 
de servicios. Informar, educar y empoderar a estos agentes es fundamental. Por otra parte, su participación es esencial, para mantener el progreso.

Un enfoque basado en los derechos, por un lado, desarrolla la capacidad de los garantes de derechos para cumplir con sus obligaciones; por otro, alienta a los titulares de derechos a reivindicarlos. Los gobiernos tienen tres niveles de obligación: respetar, proteger y hacer cumplir cada derecho. Respetar un derecho significa abstenerse de interferir en el disfrute de ese derecho. Proteger un derecho significa evitar que otras partes interfieran en ese derecho. Hacer cumplir un derecho significa adoptar medidas activas para poner en práctica leyes, políticas y procedimientos, incluida la asignación de recursos, que permitan a las personas disfrutar de los mismos.

\section{Materiales y métodos}

La investigación es de tipo cuantitativa, posee un alcance descriptivo. El muestreo que se empleó en esta investigación fue un aleatorio simple estratificado ya que se buscó otorgar énfasis y equidad en la distribución de los y las estudiantes encuestados por carrera. La ecuación de muestreo fue:

$$
n=\frac{Z_{\alpha / 2}^{2} P Q N}{\varepsilon^{2}(N-1)+Z^{2} P Q}
$$

Donde:

$Z=1.96$

$e=0.05$

$P=0.5$

$Q=0.5$

$\mathrm{N}=10.774$

Se obtuvo una muestra de 371 estudiantes, pero con el afán de mejorar la calidad y confiabilidad de los resultados se planteó una meta de al menos 500 encuestas. Finalmente terminaron lográndose 876 cuestionarios; lo que implica que con este tamaño de muestra se alcanza mayor confiabilidad en la normalidad de los resultados.

Para describir "con qué", es decir los insumos, materiales, equipos, etc., necesarios para implementar la investigación, a continuación se sintetiza las sugerencias que plantea Di Virgilio and Solano (2012) : 


\section{Encuestas}

Se aplicó el cuestionario impreso vía digital a los y las estudiantes luego de haber realizado una muestra estadística para todas las carreras y se contó con un experto de estadística para calcular la muestra.

El cuestionario se realizó a través de una plataforma web, simplemente se pidió apoyo a los y las estudiantes con lista de correos electrónicos a los que fue enviado, se validó la existencia de particularidades culturales o de dialecto, se pudo apoyar con estudiantes de la carrera de Trabajo Social con la finalidad de dar seguimiento al llenado de las encuestas y aplicación del cuestionario.

\section{¿Qué recursos se utilizaron?}

- Humanos. La preparación de una encuesta requiere tiempo y previsión por parte del/de la encuestado/a. Además, la sistematización de los datos pudo demandar una dedicación mayor. Hay que tener en cuenta que el número de encuestas y que es importante que domine algunas técnicas (capacidad del manejo tecnológico).

- Tecnológicos Para registrar las encuestas es necesario contar con un computador o con un celular de alta generación.

- Presupuestarios Los gastos de la encuesta son mínimos.

\section{Lista de control con preguntas}

- Detallar los ítems que serán monitoreados (Escala de gencat(tod@s) escala de Istas 21 para medir riesgos psicosociales solo a docentes, administrativos y trabajadores).

- Redactar preguntas que puedan ser respondidas con "sí" o "no" para cada uno de los ítems.

- Consensuar los ítems seleccionados con todos los actores que harán uso de la lista de control.

\section{Resultados}

En cuanto a lo que se refiere los datos de la encuesta aplicada se elaboraron algunas preguntas, entre ellas.

¿Cuál es su rango de edad? 


\section{Análisis cuantitativo}

La edad de las/os estudiantes de la Universidad Nacional de Loja, el $87.4 \%$ se encuentra entre el rango de $25-30$ años; un $0.5 \%$ menor a 20 años; y el resto de la muestra está en tres 30 y más años.

\section{Análisis cualitativo}

Es importante resaltar que en la institución existe población estudiantil menor a 20 años que podría en algún momento convertirse en vulnerable.

¿Seleccione a que género pertenece?

\section{Análisis cuantitativo}

Las/os estudiantes en la UNL en un $66,5 \%$ son de sexo femenino; mientras que el $33,5 \%$ son masculino; cabe señalar, se evidencia accesibilidad de las mujeres para realizar los estudios universitarios es mayoritaria.

\section{¿Selecciones su inclinación sexual?}

\section{Análisis cuantitativo}

La Inclinación sexual en las/os estudiantes de la UNL, se evidencia 97,3\% declaran ser heterosexuales (gusto por el sexo contrario); mientras que, el resto de la población analizada señala otras orientaciones sexuales.

\section{Análisis cualitativo}

Este análisis nos conlleva a deducir que el 2,97\% de los que se declaran tener afinidad por el mismo sexo, si bien es un porcentaje minino se podría inducir que este grupo se encuentre en estado de vulnerabilidad en lo que se refiere a derechos.

\section{Análisis cuantitativo}

\section{¿Indique a que facultad de la Universidad Nacional de Loja, pertenece?}

La Facultad de la Salud Humana tiene un 33,2\%, seguido de la Facultad Jurídica Social y administrativa con $26,3 \%$, la Facultad de Educación el Arte y la Comunicación un 18, 2\%, la Facultad Agropecuaria 15,2\%, la Unidad de Educación a Distancia 6,6\% y por último con un $0,3 \%$ la Facultad de la Energías y las Industrias. 
Tabla 1. Porcentajes de los estudiantes de las diferentes facultades de la Universidad Nacional de Loja

\begin{tabular}{l|l|l|l|l|l|}
$\begin{array}{l}\text { Facultad } \\
\text { de la salud } \\
\text { humana }\end{array}$ & $\begin{array}{l}\text { Facultad } \\
\text { jurídica social } \\
\mathbf{y} \\
\text { administrativa }\end{array}$ & $\begin{array}{l}\text { Facultad de la } \\
\text { educación el } \\
\text { arte y y la }\end{array}$ & $\begin{array}{l}\text { Facultad } \\
\text { agropecuaria } \\
\text { comunicación } \\
\text { y de recursos } \\
\text { naturales } \\
\text { renovables }\end{array}$ & $\begin{array}{l}\text { Unidad de } \\
\text { educación } \\
\text { a } \\
\text { distancia }\end{array}$ & $\begin{array}{l}\text { Facultad } \\
\text { de le la } \\
\text { energía y } \\
\text { las } \\
\text { industrias } \\
\text { y los } \\
\text { recursos } \\
\text { naturales } \\
\text { no } \\
\text { renovables }\end{array}$ \\
\hline $33,2 \%$ & $26,3 \%$ & $18,2 \%$ & $15,2 \%$ & $6,6 \%$ & $0.3 \%$ \\
\hline
\end{tabular}

Análisis cualitativo

La participación se midió por porcentajes de acuerdo al nivel de participación de las diferentes facultades de la Universidad Nacional de Loja, por tales razones se evidencia el nivel de participación.

\section{¿Estoy satisfecha/o con mi vida presente?}

\section{Análisis cuantitativo}

El nùmero 1 representa con el porcentaje más bajo con un 2,3\%, seguido de la escala 2 , con un $10,61 \%$, la escala 3 con un $33,78 \%$ y con la escala de 4 , un porcentaje del $53,19 \%$.

Tabla 2. Estoy satisfecha/o con mi vida presente

\begin{tabular}{l|l|l|l}
$\mathbf{1}$ & $\mathbf{2}$ & $\mathbf{3}$ & $\mathbf{4}$ \\
\hline $2,39 \%$ & $10,61 \%$ & $33,78 \%$ & $53,19 \%$
\end{tabular}

Análisis cualitativo

Para medir la satisfacción de esta pregunta fue mediante la escala, que representa 1 la calificación más baja y cuatro con la más alta. Por lo tanto se contrasta que la mitad de los encuestados se siente satisfechos con su vida presente, por otro lado la otra mitad se puede decir que hay una inconformidad. 
¿Dimensiones del estudio basados en la escala GENCAT?

Análisis Cuantitativo

Resultados descriptivos

\begin{tabular}{l|l|l|l|l}
\hline Dimensión & Media & $\begin{array}{l}\text { Calificación } \\
\text { más alta }\end{array}$ & $\begin{array}{l}\text { Desviació } \\
\text { n } \\
\text { estándar }\end{array}$ & Percentil \\
\hline Bienestar físico & 4,0 & 14 & 2,4 & 6,3 \\
\hline Autodeterminación & 9,7 & 15 & 1,3 & 46,3 \\
\hline Inclusión social & 6,5 & 17 & 2,3 & 17,0 \\
\hline Derechos & 3,3 & 13 & 1,9 & 3,2
\end{tabular}

\section{Análisis cualitativo}

Dentro de las dimensiones de la escala de GENCAT analizadas se puede evidenciar que las/os estudiantes de la UNL, la mayor dificultad se evidencia en la dimensión de derechos, es decir; si conocen sus derechos o no los conocen y que eso evidencia una vulneración de sus capacidades a decir en su vida cotidiana lo que quiere o lo desearía hacer en su vida cotidiana con un percentil de calidad de vida de 3,2 basado en los indicadores de intimidad, respeto, conocimiento y ejercicio de derechos; asimismo, seguido de bienestar físico percentil de calidad de vida de 6,3, hace referencia a tener buena salud, sentirse en buena forma física, tener hábitos de alimentación saludables, cuyos indicadores son: atención sanitaria, sueño, salud y sus alteraciones, actividades de la vida diaria, acceso a alimentación; generalmente está dimensión es bastante preocupante en la comunidad estudiantil, por cuanto su estado físico y de salud integral no es el normal ni el adecuado.

Las dimensiones de autodeterminación e inclusión social presentan resultados en referencia a:

Autodeterminación entendida está por decidir por sí mismo y tener oportunidad de elegir las cosas que quiere, cómo quiere que sea su vida, su trabajo, su tiempo libre, el lugar donde vive, las personas con las que está, los resultados son percentil de calidad de vida de 46,3; inclusión se midió con los indicadores de integración, participación, accesibilidad y apoyos percentil de 17, éstas dimensiones han generado analizar en concordancia con los referentes teóricos de vulnerabilidad social se entiende como la incapacidad de una persona para aprovechar las oportunidades, disponibles en distintos ámbitos socioeconómicos, para mejorar su situación de 
bienestar o impedir su deterioro suelen desencadenar sinergias negativas que tienden a un agravamiento progresivo.

\section{Conclusiones}

A medio término de la investigación se establecen las siguientes conclusiones:

La investigación permitió analizar las condiciones personales, colectivas y sociales que tienen las personas específicamente los y las estudiantes de la Universidad Nacional de Loja, en su situación porque carecen de múltiples necesidades, porque tienen limitados recursos económicos que no les permite solventar sus necesidades personales y de sus familias. La calidad de vida de los y las estudiantes que llevan no es la adecuada debido a que existen ciertos factores que impiden que estas personas se desenvuelvan en su contexto social de manera idónea como por ejemplo alimentarse bien, ejercer el derecho a la educación y la salud, porque algunos desconocen sus derechos sexuales y reproductivos perjudicando su estilo de vida.

Es importante trabajar y desarrollar procesos de iniciativas y técnicas o actividades en la búsqueda del involucramiento de la comunidad universitaria, en este caso de los y las estudiantes, mejorar su autoestima y especialmente trabajar en temas de participación y cooperación, diseñando proyectos que mejoren la percepción y las condiciones de vida en las que se encuentran actualmente, establecer mecanismos de seguimiento que velen por mejorar las condiciones, y pueda desarrollar sus facultades plenamente en un ambiente idóneo para cumplir sus objetivos.

Con ésta investigación se puede identificar la necesidad de que la Universidad institucionalice el tema de participación y derechos con la finalidad que sean temas que se discuten en varios espacios por lo que hay la posibilidad de transversal izar en las funciones específicas de las instituciones de educación superior para poder discutir de estos temas en la Docencia, en la Investigación, en Vinculación con la colectividad, y gestión administrativa, creando proyectos que conlleven a brindar oportunidades del ejercicio de derechos en todas las funciones sustantivas que realiza la Universidad.

Se recomienda utilizar como herramienta metodológica a la escala de GENCAT para evaluar constantemente a través de indicadores la calidad de vida de las personas que se encuentra inmersas en situaciones de vulnerabilidad, puesto que es importante para el desarrollo de la sociedad y para proporcionar información confiable y segura acerca de las mismas de tal manera que las autoridades pueda tomar decisiones frente a la realidad local. El enfoque de derechos puede ser abordado desde diferentes puntos de vista. Desde una mirada orientada hacia la intervención en lo social, con la posibilidad de comprender y explicar los problemas sociales, aportando a su vez 
líneas de análisis que logran sostener las prácticas, especialmente desde la idea de interrelación con la finalidad de tener la mirada integral del ser humano.

Fomentar redes de trabajo que involucren a la comunidad Universitaria y que fomenten la participación y el trabajo en equipo, tiene muchas ventajas: hablar, escuchar, conocer, intercambiar, aprender y resolver problemas en conjunto, desarrollar capacidades, enriquecer la comprensión de los fenómenos, ampliar la mirada holística, y, por lo tanto, mejorar los servicios de atención a la comunidad universitaria.

\section{Referencia}

Alonso, V. (Enero de 2009). MANUAL DE APLICACIÓN DE LA ESCALA DE GENCAT DE CALIDAD DE VIDA. 80 páginas. Barcelona.

Ángel, V. A. (s.f.). Escala GENCAT: manual de aplicación de la Escala GENCAT de Calidad de vida. 2009, 80. Barcelona.

Bravo, R. (2000). Condiciones de Vida y Desigualdad Social. CEPAL.

CINTERFOR. (2012). Guia para la evaluación de Indicadores. Obtenido de http://guia.oitcinterfor.org/como-evaluar/como-se-construyen-indicadores

Coello, J. (2005). ANÁLISIS ESTADÍSTICO-DISUR Población Nacional Según Grupos de edad. Loja. Obtenido de https://es.slideshare.net/andfer03/indicadores-sociales

Gálves P, T. (2001). Aspectos Económicos de la equidad de género. Santiago de Chile: CEPAL-ECLAC.

Garriga, Y., Navarro, J., \& Saumell, A. (2008). Determinantes de la salud: el rol de la inequidad en salud. Obtenido de http://bvs.sld.cu/revistas/infd/n1512/infd07212.htm

Kaztman, R. (2000). Documentos de Trabajo IPES. 2(2), 40. Uruguay.

Labrunée, M. E., \& Gallo, M. (2005). Vulnerabilidad Social: El Camino Hacia la Exclusión. (T. d. local, Ed.) Mar de la plata: Suárez. Obtenido de http://nulan.mdp.edu.ar/id/eprint/716

MIGUEL, A. (s.f.). RESILIENCIA: OTRA PERSPECTIVA DE LAS EXPERIENCIAS SOCIALES Y PERSONALES DENTRO DE LA EMPRESA. Estudios Gerenciales, 9.

Mokate, K. (2002). EFICACIA, EFICIENCIA, EQUIDAD Y SOSTENIBILIDAD: ¿QUÉ QUEREMOS DECIR? INDES. 
Mosquera, N., Rengifo, N., Vidal, Z., \& Menjura, M. (2013). Análisis de factores familiares y sociales asociados a vulnerabilidad. Plumilla Educativa, 26.

Romero, P. (03 de Sep de 2016). Noti mundo. Obtenido de El fenómeno de la migración en Ecuador: https://notimundo.com.ec/el-fenomeno-de-lamigracion-en-ecuador/

Labrunée , M. E., \& Gallo, M. (2005). Vulnerabilidad Social: El Camino Hacia la Exclusión. Facultad de Ciencias Económicas y Sociales, Universidad Nacional de Mar del Plata, 21.

Alonso, V. (Enero de 2009). MANUAL DE APLICACIÓN DE LA ESCALA DE GENCAT DE CALIDAD DE VIDA. 80 páginas. Barcelona.

Ángel, V. A. (s.f.). Escala GENCAT: manual de aplicación de la Escala GENCAT de Calidad de vida. 2009, 80. Barcelona.

Bravo, R. (2000). Condiciones de Vida y Desigualdad Social. CEPAL.

CINTERFOR. (2012). Guia para la evaluación de Indicadores. Obtenido de http://guia.oitcinterfor.org/como-evaluar/como-se-construyen-indicadores

Coello, J. (2005). ANÁLISIS ESTADÍSTICO-DISUR Población Nacional Según Grupos de edad. Loja. Obtenido de https://es.slideshare.net/andfer03/indicadores-sociales

Gálves P, T. (2001). Aspectos Económicos de la equidad de género. Santiago de Chile: CEPAL-ECLAC.

Garriga, Y., Navarro, J., \& Saumell, A. (2008). Determinantes de la salud: el rol de la inequidad en salud. Obtenido de http://bvs.sld.cu/revistas/infd/n1512/infd07212.htm

Kaztman, R. (2000). Documentos de Trabajo IPES. 2(2), 40. Uruguay.

MIGUEL, A. (s.f.). RESILIENCIA: OTRA PERSPECTIVA DE LAS EXPERIENCIAS SOCIALES Y PERSONALES DENTRO DE LA EMPRESA. Estudios Gerenciales, 9.

Mokate, K. (2002). EFICACIA, EFICIENCIA, EQUIDAD Y SOSTENIBILIDAD: ¿QUÉ QUEREMOS DECIR? INDES.

Mosquera, N., Rengifo, N., Vidal, Z., \& Menjura, M. (2013). Análisis de factores familiares y sociales asociados a vulnerabilidad. Plumilla Educativa, 26.

Romero, P. (03 de Sep de 2016). Noti mundo. Obtenido de El fenómeno de la migración en Ecuador: https://notimundo.com.ec/el-fenomeno-de-la-migracionen-ecuador/ 